\title{
Characterisation of Regional Variations in a Stitched CMOS Active Pixel Sensor
}

\author{
Hafiz M Zin ${ }^{1 *}$, Anastasios C Konstantinidis ${ }^{2}$, Emma J Harris ${ }^{1}$, John FP Osmond ${ }^{1}$, \\ Alesandro Olivo ${ }^{2}$, Sarah E Bohndiek ${ }^{2}$, Andy T Clark ${ }^{3}$, Renato Turchetta ${ }^{3}$, \\ Nicola Guerrini ${ }^{3}$, Jamie Crooks ${ }^{3}$, Nigel M Allinson ${ }^{4}$, Robert Speller ${ }^{2}$ and Philip M Evans ${ }^{1}$ \\ ${ }^{1}$ Joint Physics Department, Institute of Cancer Research and Royal Marsden NHS \\ Trust, Downs Road, Sutton, Surrey, SM2 5PT, UK \\ ${ }^{2}$ Dept. Medical Physics and Bioengineering, Malet Place Engineering Building, \\ University College London, Gower Street, London, WC1E 6BT, UK \\ ${ }^{3}$ Rutherford Appleton Laboratory, Chilton, Didcot, Oxfordshire, OX11 0QX, UK \\ ${ }^{4}$ Department of Electronic \& Electrical Engineering, University of Sheffield, Mappin \\ Street, Sheffield, S1 3JD, UK \\ *Author for correspondence: hafiz.zin@icr.ac.uk
}

\begin{abstract}
Stitched, large area, complementary metal-oxide-semiconductor (CMOS), active pixel sensors (APS) show promise for x-ray imaging applications. In this paper we present an investigation of the effects of stitching on uniformity of sensor response for an experimental APS. The sensor, known as LAS (large area sensor), was made by reticular stitching onto a single silicon wafer of a 5 by 5 array of regions consisting of $270 \times 27040 \mu \mathrm{m}$ pixels to yield $1350 \times 1350$ pixels and an imaging area of $54 \mathrm{~mm} \times$ $54 \mathrm{~mm}$. Data acquired from two different sensor of the same type were filtered to remove spiking pixels and electromagnetic interference (EMI). The non-linear compensation technique for CMOS sensor analysis was used to determine the variation in gain, read noise, full well capacity and dynamic range between stitched regions. Variations across stitched regions were analysed using profiles, analysis of pixel variations at stitch boundaries and using a measurement of non-uniformity within a stitched region. The results showed that non-uniformity variations were present, which increased with signal (1.5-3.5\% at dark signal, rising to 3-8\%). However these were found to be smaller than variations caused by differences in readout electronics, particularly at low signal levels. The results suggest these variations should be correctable using standard calibration methods.
\end{abstract}

Keywords: complementary metal-oxide-semiconductor, active pixel sensor, wafer scale sensor, reticle stitching 


\section{Introduction}

Flat panel imagers (FPIs) have gained popularity for medical x-ray imaging [1] due to their large image area, which is typically greater than $20 \mathrm{~cm} \times 20 \mathrm{~cm}$. Medical x-ray applications include fluoroscopy [2] and radiotherapy motion tracking [3] which generally require a large format sensor and near real-time frame rates (between $10-$ $50 \mathrm{~s}^{-1}$ ). The faster frame rates present a challenge for large format sensors.

Other sensors technologies such as charge coupled devices (CCD) and complementary metal-oxide-semiconductor (CMOS) active pixel sensors (APS) have superior performance in terms of fast read-out, low noise and high spatial resolution compared to FPIs. APSs allow row and column addressing for random pixel access and on-chip fast multiple region-of-interest read-out. This functionality allows the user to trade resolution, or array size, with read-out speed. In addition, shrinking transistor sizes means that more functionality can be built into the pixel, i.e. in-pixel analogue-to-digital conversion or in-pixel memory [4]. Despite these advantages, until very recently, their small size has limited their application to medical imaging. Previously, individual CCDs have been tiled together, to form large format devices, initially using mosaics of devices that were slightly overlapping [5]. Later buttable devices were designed and these sensors were manufactured so that the read-out electronics remained only on one or two sides of the sensor, therefore allowing them to be placed in the same plane, reducing the amount of dead space between them. Fully buttable CCDs are now available.

More recently photolithographic techniques have advanced to enable the creation of large format devices that are integrated onto a single silicon wafer to form a contiguous sensor array. By stepping a reticle across a wafer of silicon, smaller arrays can be "stitched" together to construct larger area sensors. Both large area CCDs and APSs have been manufactured in this way.

One of the challenges with large format sensors is maintaining spatial uniformity across the focal plane. For tiled devices comprising of multiple sensors, individual sensor characteristics will vary due to differences in noise and device sensitivity. Careful calibration of large area devices is required to form a correction map to achieve uniform imaging response. The application of such a correction map is relatively straightforward, however large non-uniformities will result in the reduction of dynamic range of the sensor. Despite new large area sensors being monolithic, sources of non-uniformity persist. These can include spatial variations across the silicon wafer caused by manufacturing variations, including defects in the wafer, amplifier variations and variations between read-out electronics. Variation might also result from slight imperfection between stitched regions.

This paper describes an investigation into the spatial non-uniformity of a sensor manufactured using reticular stitching. This paper presents an investigation into the sources of electronic noise and how best to remove them. In the absence of these noise sources, read noise, sensor gain (at low signal level and high signal level), dynamic range and full well capacity have been investigated for each of the stitched regions and the variation between them quantified. In addition fixed pattern noise within the stitched regions and across the whole sensor array has been measured for several illumination levels. 


\section{Materials and methods}

\subsection{Large area sensor (LAS) description}

A new monolithic APS has been developed by the Multidimensional Integrated Intelligent Imaging (MI3) consortium (RC-UK Basic Technology Programme) [6,7]. This APS, named Large Area Sensor or LAS, was constructed by stitching together a $5 \times 5$ array of pixel regions on a single wafer, and fabricated using a $0.35 \mu \mathrm{m}$ CMOS process $[7,8]$.

The architecture of LAS is shown in figure 1. The sensor array was constructed by stepping a sub-block mask of $10.8 \mathrm{~mm} \times 10.8 \mathrm{~mm}$ optically across the wafer [9]. The sub-block mask formed a $270 \times 270$ pixel array (labelled D in figure 1 ). It was stepped and imaged 25 times to form the $5 \times 5$ array of "stitched regions" which contained a total of $1350 \times 1350$ pixels or 1.8 Megapixels with an imaging area of 54 $\mathrm{mm} \times 54 \mathrm{~mm}(58 \mathrm{~mm} \times 58 \mathrm{~mm}$ die size $)$. The sensor was completed with the assorted connection pads and read-out electronics in the corner $(\mathrm{A})$ and the edge pieces $(\mathrm{B}, \mathrm{C})$. Each block $\mathrm{C} 1$ on the left contained 270 row decoders and drivers. The reset circuitry was placed in block B1 on the top while the read-out circuitry was located in block B2 at the bottom. Two examples of the sensor were used in this work which we refer to as LAS 1 and LAS 2.

Pixels were read out in rolling shutter mode on a row by row basis using two sampling capacitors located at the end of each column. The imager has 10 differential parallel analogue output channels which are 135 pixels wide. Each of these output channels is referred to as a sub-column. Pixel read-out for every sub-column was converted by one of 10 off-chip Analogue to Digital Converters (ADC). The ADCs used were 14-bit, pipelined, model AD1252 ADC running at 5M samples per second (Analog Devices Inc., Norwood, MA, USA). For a detailed description of the pixel architecture, the reader is referred to [7]. We refer to the 5 columns of the stitched region array as columns $1,2 \ldots$ etc and to the 10 sub-columns as $1 \mathrm{a}, 1 \mathrm{~b}, 2 \mathrm{a}, 2 \mathrm{~b} \ldots$ etc (Fig. 1).

The sensor was mounted on a printed circuit board (PCB) based Chip On Board (COB) custom package and connected to a stack of circuit boards via three headers. The stack consisted of the ADCs, the connection to the power supply and a field programmable gating array (FPGA). The OptoDAQ data acquisition system, (Rutherford Appleton Laboratory, Didcot, Oxford, UK) was centred around the FPGA board where the control signals required to drive the sensor were generated. The acquired 14-bit digitised images were then transferred to a computer via a fiber optic link. Image acquisition was controlled using C-based software. Full frames were read out at a rate of 10 frame $\mathrm{s}^{-1}$. All image processing was carried out using MATLAB (The Mathworks Inc., Natick, MA, USA).

\subsection{Characterisation of electronic noise sources}

It has been shown that CMOS APSs can be affected by noise sources in addition to those seen with CCDs, including random telegraphic noise [10]. These noise sources 
primarily dominate the pixel read noise floor in CMOS imagers [11]. Previous work [12] has shown that at low signal levels, LAS is dominated by a strong columnar pattern due to variations in electronic components that read out signal from different columns of the device. Prior to spatial uniformity analysis of the sensor array it was necessary to characterise these sources of noise in order to determine how best to remove them.

Figure 2 shows a dark image acquired using LAS 1 which is the average of 200 frames. Columnar variation due to non-uniformity between the read-out electronics dominates the dark signal making it harder to distinguish the $5 \times 5$ stitched regions. This pattern shows the 10 sub-columns, each corresponding to one of the 10 ADCs as each stitched region is read out by two ADCs.

To determine the variation in the magnitude of dark noise between sub-columns we acquired 100 consecutive dark frames and determined the variation (standard deviation (SD)) in the dark signal (measured in digital numbers or DN) for each pixel. We found the distribution of pixel dark noise for pixels within each of the subcolumns. The shape of the distribution for 6 sub-columns (1a, 1b, 2b, 3b, 4b and 5b) was found to be skewed towards high SD values, indicating that a number of pixels have higher than expected SD; we would expect SD values would be normally distributed. Visual examination of the images revealed that a number of pixels in these sub-columns contained pixels which switched from low to high pixel values between frames. We refer to these as spiking pixels. Figure 3 shows a plot of the SD of pixel values as a function of frame number for an example of these sub-columns, $3 \mathrm{~b}$. High SD corresponds to the presence of spiking, i.e. the presence of high pixel values increases the SD. Spiking is almost periodic with typically 8 or 9 good frames followed by 9 or 10 frames with high SD. This periodic behaviour was observed for sub-columns1b, 2b, 3b, 4b and 5b. Sub-column 1a showed high SD for all frames. We will refer to the frames containing spiking pixels as spiking frames ( $\mathrm{Sp}$ frames) and those without as non-spiking frames (NSp frames). Another source of spatial variation observed in the dark images was due to electromagnetic interference or EMI [13]. The frequency of the EMI was calculated to be $\sim 200 \mathrm{kHz}$ and originates from the stack of boards itself.

\subsection{Removal of electronic noise sources}

The frames with spiking in sub-columns $1 \mathrm{~b}, 2 \mathrm{~b} \ldots 5 \mathrm{~b}$ were excluded from the analysis of the effects of stitching. Spiking was present in sub-column 1a for all frames and there was some level of spiking in sub-column $5 b$ for all frames.

EMI noise was filtered out from the frames using a frequency-space filter method [13]. Briefly, all frames were filtered using a top-hat filter in the Fourier domain to detect frequencies with high power. These frequencies were removed using a crossshaped Gaussian notch reject filter [14].

We evaluated the various filters by analysing the coefficient of variation (COV) of the pixel dark noise in each of the sub-columns for 5 cases: all frames without EMI filtration; spiking frames only without EMI filtration; non-spiking frames only without EMI filtration; spiking frames with EMI filtration, and non-spiking frames with EMI filtration. COV is the ratio of the standard deviation to the mean. 


\subsection{Evaluation of sensor performance parameters}

Optical characterisation of the sensor's photon transfer curve was carried out using the Non-Linear Compensation (NLC) technique described by Janesick [15]. Recently the use of this method to evaluate the whole LAS sensor has been reported [8]. Variations between stitch regions will be presented in this report.

In brief, the optical set-up used was based upon recommendations from the EMVA Standard for characterisation and presentation of specification data for image sensors and cameras [16]. Both sensors were uniformly illuminated using varying light intensity from zero (dark image) to an intensity which saturated the sensor. Prior to analysis, images were corrected for the electronic noise sources discussed in section 2.3.

Characteristics measured were: read noise floor (in electrons); noise gain (electrons per DN); signal gain (electrons per DN); full well capacity (electrons), and dynamic range $(\mathrm{dB})$. Read noise floor and full well capacity were measured as DN and converted into units of electrons using noise and signal gain values respectively. Full well capacity was determined from the signal level at which image noise began to reduce due to pixel saturation. Dynamic range was defined as full well capacity divided by the read noise floor.

\subsection{Evaluation of variations between stitched regions}

In order to enable evaluation of the variations between stitched regions, a set of frames was acquired for dark signal, low signal (in the region where read noise dominates) and half saturation signal (where shot noise dominates). For each signal level, a set of 28 frames without spiking was selected and averaged. Unless otherwise stated, residual sub-columnar variation in the dark signal was corrected for by multiplying the signal in sub-column $\mathrm{i}$ by a field correction factor $\mathrm{FF}_{\mathrm{i}}$, where $\mathrm{FF}_{\mathrm{i}}$ is equal to the mean dark signal $\left(D_{S}\right)$ in sub-column i divided by the mean value of $D_{S}$ for the whole sensor.

The variations between stitched regions were evaluated by measuring deviations from row-to-row (top to bottom in Fig. 2). Deviations from column-to-column were dominated by variations in the readout channels as discussed above and not analysed further. Three analyses were carried out.

Firstly, for the dark signal data, row-to-row profiles (along the column) were acquired and averaged for each of the 25 sub-regions. Each profile had a 7 element rolling average filter applied to reduce noise. In addition the row-to-row profile (along the column), averaged over all columns in the sensors was calculated. The second analysis aimed to determine the percentage difference at the boundaries between stitched regions, averaged over all columns for the dark signal data. The third analysis aimed to calculate the size of the non-uniformity in the stitched regions. For the rowto-row profile, averaged over all columns, the maximum (max) and minimum (min) values were determined and the percentage non-uniformity was calculated using eq. 1 : 


$$
\text { Non - uniformity }(\%)=\frac{\max -\min }{\max +\min } \times 100
$$

Values were analysed for each of the three signal levels.

\section{Results and discussion}

\subsection{Removal of electronic noise sources}

To evaluate the effects of using the various filter methods on pixel dark noise, the average pixel noise value for each of the 10 sub-columns was determined and the $\mathrm{COV}$ between the regions evaluated. This is shown in figure 4 for each of the five cases. The periodic high SD frames (case 2) are believed to coincide with the frame transfer data burst between the FPGA and the PC. The $\sim 200 \mathrm{kHz}$ noise is believed to come from the $-5 \mathrm{~V}$ power supply within the DAQ system. Therefore both noise sources are probably an artefact of the DAQ system not the sensor. As expected, the combination of non-spiking frames and EMI filter gave the smallest COV. Figure 5 shows maps of the mean pixel dark noise for each of the sub-stitched regions (i.e. the 50 regions defined by the stitch rows and the sub-columns). It can be seen in Fig 5 ('Sp' and 'Sp+EMIf' maps) that EMI filtration has the effect of increasing the subcolumnar variation. This suggests that the EMI filter results in artefacts in the presence of spiking pixels. From Fig 4 the application of EMI filtration and the selection of non-spiking frames reduces the coefficient of variation however some residual sub-column variation remains (Fig 5). A skewed distribution of pixel SDs, as caused by the spiking pixels, is characteristic of Random Telegraph Signal (RTS) noise [10,11]. However the periodic nature (Fig. 3) suggests interference as the source.

\subsection{Variation in performance parameters across stitched regions}

Figure 6 shows the results of the analysis using the NLC technique for LAS 1. Maps are shown of the following quantities: dark signal $\left(\mathrm{D}_{\mathrm{S}}\right)$, noise gain $(\mathrm{N})$, signal gain (S), read noise $(\mathrm{RdN})$, full well capacity $(\mathrm{FW})$, and dynamic range (DR). Each map shows the mean value in each of the sub-stitch regions expressed as a percentage of the mean value of all sub-stitch regions.

The gain parameters, $\mathrm{S}$ and $\mathrm{N}$, are high in column 1, due to residual spiking pixels. S, $\mathrm{N}$ and FW appear to decrease from the top left hand corner to the bottom right corner suggesting structure in the sensor. The differences between the pairs of sub-columns are generally small in these plots except for column 1. Also RdN is highest in subcolumn 1, again due to the presence of spiking pixels in this sub-column for all frames. Dynamic range is uniform across the sensor. 
Table 1 presents numerical values for the sensor characterisation shown in Fig 6. For each of the six parameters, the average value across the sensor is shown along with COV values across row-by-row and column-by-column directions. In table 1, values for sub-column 1a are excluded as this showed behaviour substantially different from the rest of the sensor, due to the presence of spiking pixels in all frames. Row-by-row $\mathrm{COV}$ values were obtained by calculating the COV for sub-column $1 \mathrm{~b}, 2 \mathrm{a} \ldots 5 \mathrm{~b}$ and averaging their values. A similar procedure was used for the column-by-column COV analysis. In all cases the row-by-row values are smaller, indicating the dominance of the variations between sub-columns. The results for the mean values for the sensor parameters are comparable to those presented by Bohndiek et al. (2009) [8]. However, the values presented by Bohndiek are for a ROI within a central sub-column, and with the presence of spiking pixels and without EMI corrections.

\subsection{Fixed pattern noise variations between stitched regions}

Figure 7 shows the average row-to-row profile plots for each of the stitched regions for LAS 1 . The plots have a distinctly parabolic pattern which varies from left to right across the graph (row block 1 to row block 5, where each of the 5 row blocks comprises 270 rows). There are also discontinuities at the boundaries between the stitched regions. Figure 8 shows the row-to-row profile averaged across the whole sensor for both LAS 1 and LAS 2. The parabolic pattern is observed in both of the sensors. Also the pixel-to-pixel variations are correlated in the two sensors. The greatest variation across the profiles is observed in row block 5 i.e. sensor rows 1080 to 1350 . This shows a change of $160 \mathrm{DN}$ (from $4000 \mathrm{DN}$ to $4160 \mathrm{DN}$ ). This should be compared with the dynamic range of the sensor, which is about 9500 in DN. LAS 2 had three lines of dead pixels, which precluded calculation of the profile for this sensor at three rows in Fig 8 (in row blocks 1, 2 and 4).

Figure 9 gives the percentage differences between the boundaries of the stitched regions for both sensors. Numbers shown are the modulus of the difference in pixel values either side of the boundary as a percentage of the average. Differences of 1.5$2.0 \%$ are seen. These differences at the boundaries support the hypothesis that the variation along the columns may be due to the non-uniformity in the reticle and the mask during stitching process rather than variation in the wafer quality. Figs 7 to 9 show that there is greater dark signal at one end of a stitch region than at the other (increasing from top to bottom of the sensor). Dark signal is expected to arise principally from thermal current and offset. It may be that the pixels physically change size across the stitched region. This may be a consequence of the process of reticle stitching resulting in small difference in the magnification of the projected reticle. Variations in the quality of the pixels put down by the lithographic process may be a contributing factor.

Figure 10a shows a bar chart of percentage non-uniformity as a function of row block number for both sensors at three illumination levels: dark level, low signal and half saturation signal. Average pixel values for the three cases (DN) were 4028, 5189 and 9015 and 4093, 5058, 8397 for LAS 1 and LAS 2 respectively. The saturation value for each sensor was 13445 and 12489. With the exception of one data point (row block 1 for LAS 2), the non-uniformity increases with signal suggesting that the origin of the non-uniformity may be a source other than simple offset variation and may have an effect that scales with signal. Non-uniformity values are $1.5-3.5 \%$ of 
signal for the dark level, rising to $3-8 \%$ for the higher signal. To put these nonuniformity values in the context of the dynamic range, take as example, LAS 1 which has a dynamic range of $9417 \mathrm{DN}$. The differences between minimum and maximum represented by the non-uniformity values are: 121, 211 and 902 (all DN) for dark signal, low signal and half saturation signal, respectively. Fig $10 \mathrm{~b}$ shows the corresponding non-uniformity measured in the column-to-column direct. For this data, the sub-column gain variations have not been corrected to enable a comparison between the effects of stitching and readout channel gain variations. As discussed above, the stitching variations (Fig 10a) are smaller than the sub-column variations (Fig 10b) resulting from differences in readout gain, particularly for small signal levels. The variation in readout gain is likely due to both the on-chip output amplifier and the external buffering in the DAQ system before the ADC.

\section{Conclusions}

We have investigated dark signal non-uniformity and variations in signal gain between stitched regions in a large format CMOS APSs. The purpose of this study was to investigate variations that could arise due to the stitching process used to form such large pixel arrays. The dominant sources of noise in the APS at low signal values are differences that arise from difference in the electronic components. When these were removed we observed distinct fixed pattern noise variations between the stitched regions. However, the non-uniformity arising from stitching was small in comparison to non-uniformities in read-out, particularly for small signals. They can easily be corrected for using field corrections which should only result in a small reduction in dynamic range. The fact that non-uniformities can be easily corrected for by means of field correction makes detectors like LAS even better suited for applications in medical imaging.

\section{Acknowledgements}

This work is supported by the RC-UK Basic Technology Multidimensional Integrated Intelligent Imaging (MI-3) programme (GR/S85733/01) and EPSRC grants $\mathrm{EP} / \mathrm{G} 037671 / 1$ and EP/F035985/1. HZ is funded by a grant from the Malaysian Government and Universiti Sains Malaysia. Work at ICR is partial funded by Cancer Research UK C46/A2131 and National Health Service funding to the Biomedical Research Centre. AK is supported by the Greek State Scholarships Foundation. AO is supported by a Career Acceleration Fellowship awarded by the UK Engineering and Physical Sciences Research Council (EP/G004250/1). 


\section{References}

[1] L.E. Antonuk, J. Boudry, W. Huang, K.L. Lam, E.J. Morton, R.T. Haken, J. Yorkston, N.H. Clinthorne, IEEE T Med Imaging 13 (1994) 482.

[2] A.R. Cowen, A.G. Davies, M.U. Sivananthan, Clin Radiol 63 (2008) 1073.

[3] J.F.P. Osmond, E.J. Harris, A.T. Clark, R.J. Ott, A.D. Holland, P.M. Evans, Phys Med Biol 53 (2008) 3159.

[4] R. Turchetta, et al., Nucl. Instr. Methods. A 560 (2006) 139.

[5] L. Strüder, et al., Astron Astrophys 365 (2001) L18.

[6] N. Allinson, et al., Nucl. Instr. Methods. A (2009) 196.

[7] A.T. Clark, N. Guerrini, N. Allinson, S.E. Bohndiek, J. Crooks, T. Pickering, R. Turchetta, A 54mm x 54mm - 1.8Megapixel CMOS image sensor for medical imaging, IEEE Nuclear Science Symposium Conference Record, Dresden, Germany, 2008, pp. 4540.

[8] S.E. Bohndiek, et al., IEEE T Nucl Sci 56 (2009) 2938.

[9] A. Hoffman, M. Loose, V. Suntharalingam, Exp Astron 19 (2006) 111.

[10] J.R. Janesick, J.T. Andrews, T. Elliott, Proc. of SPIE 6276 (2006).

[11] W. Xinyang, P. Rao, A.M.A. Theuwissen, Random Telegraph Signal in CMOS Image Sensor Pixels, International Electron Devices Meeting, San Francisco, CA, 2006.

[12] S.E. Bohndiek, A. Blue, A.T. Clark, M.L. Prydderch, R. Turchetta, G.J. Royle, R.D. Speller, IEEE Sens J 8 (2008) 1734.

[13] A.C. Konstantinidis, S.B. Bohndiek, A. Olivo, P.R.T. Munro, R.D. Speller, to be submitted (2009).

[14] R.C. Gonzalez, R.E. Woods, Digital Image Processing, Prentice Hall, 2008.

[15] J.R. Janesick, Photon Transfer DN $\rightarrow \lambda$ SPIE Press., 2007.

[16] EMVA Standard 1288: Standard for characterization and presentation of specification data for image sensors and cameras, European Machine Vision Association, 2005. 



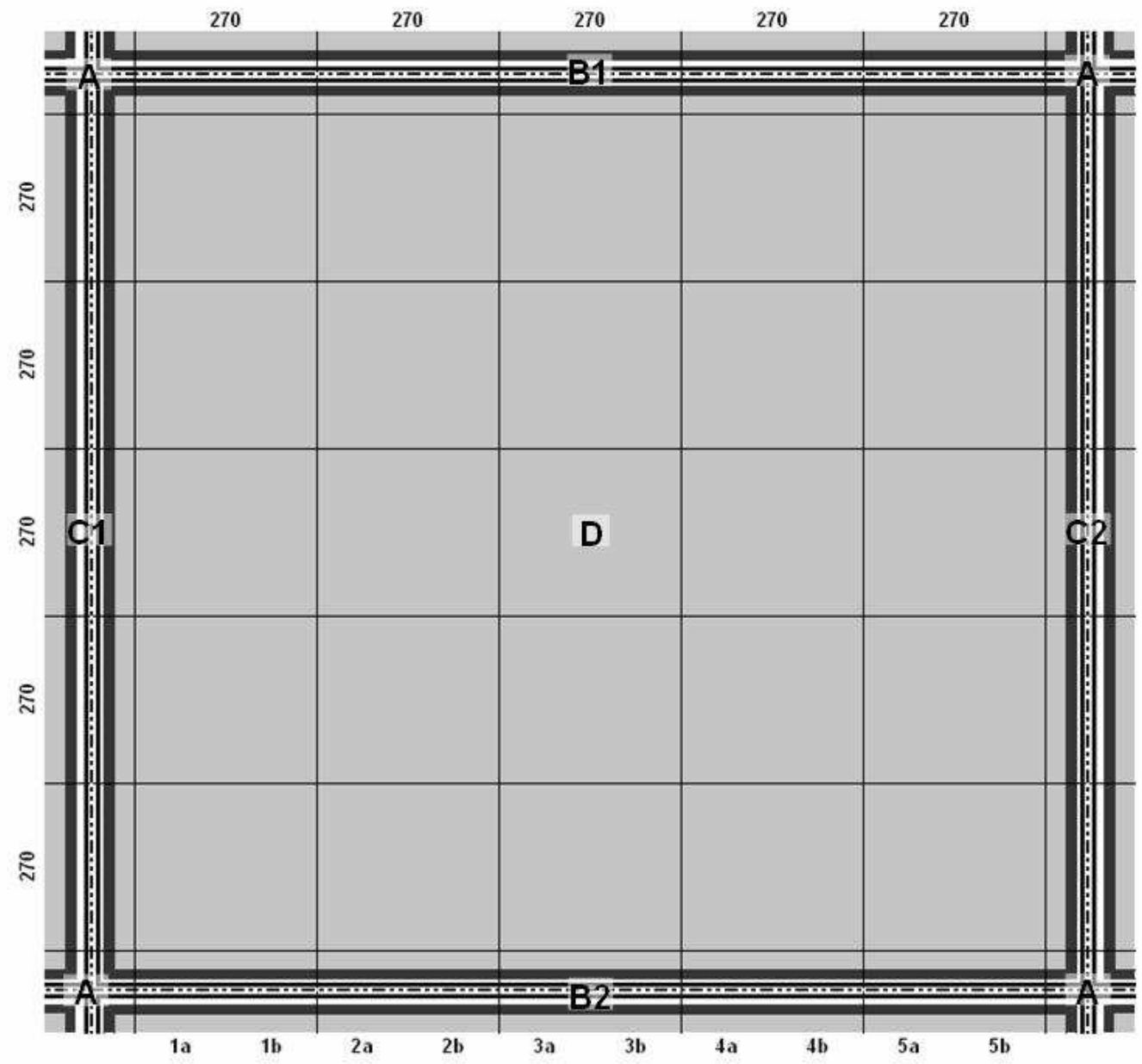

Figure 1 


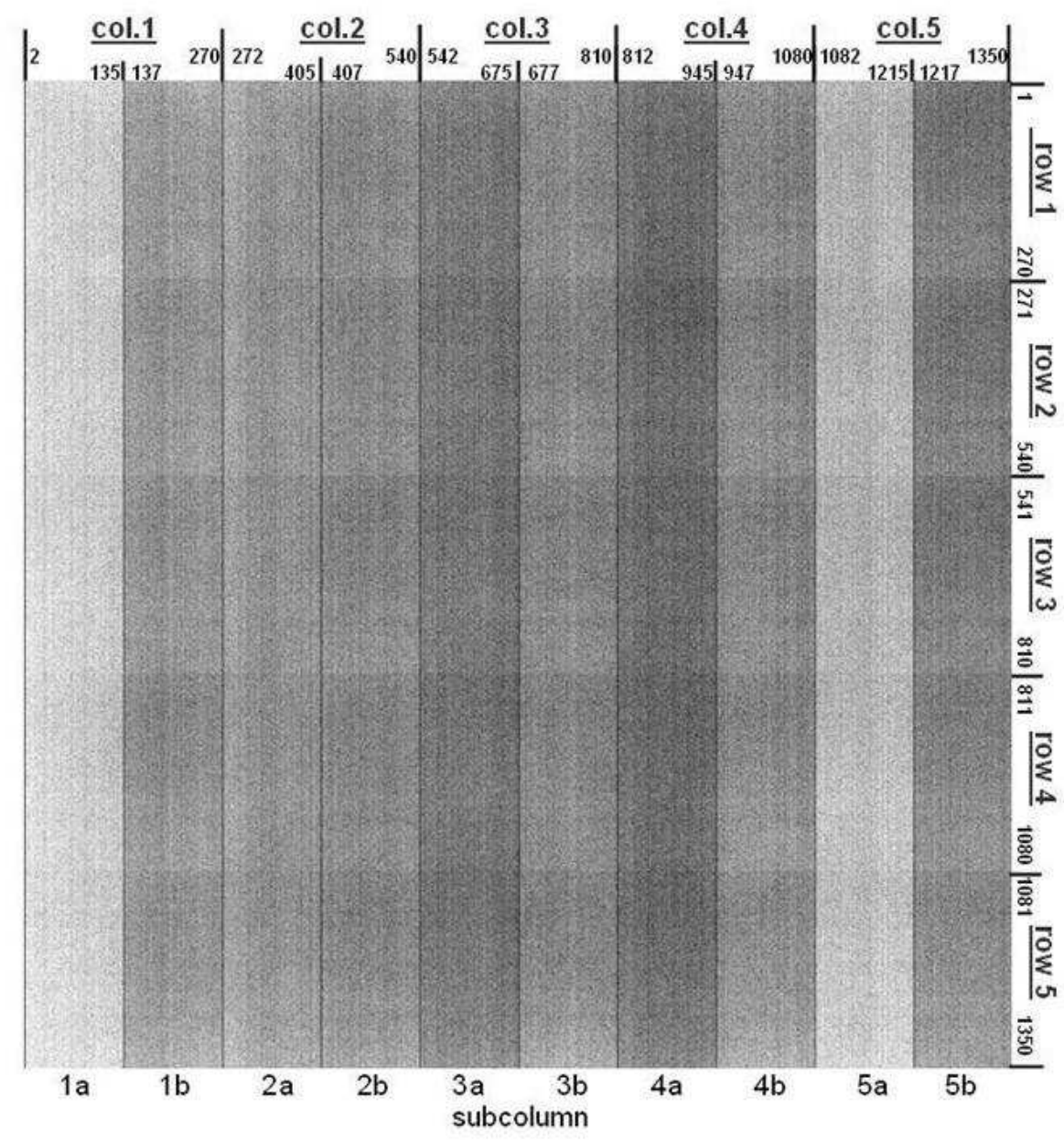

Figure 2

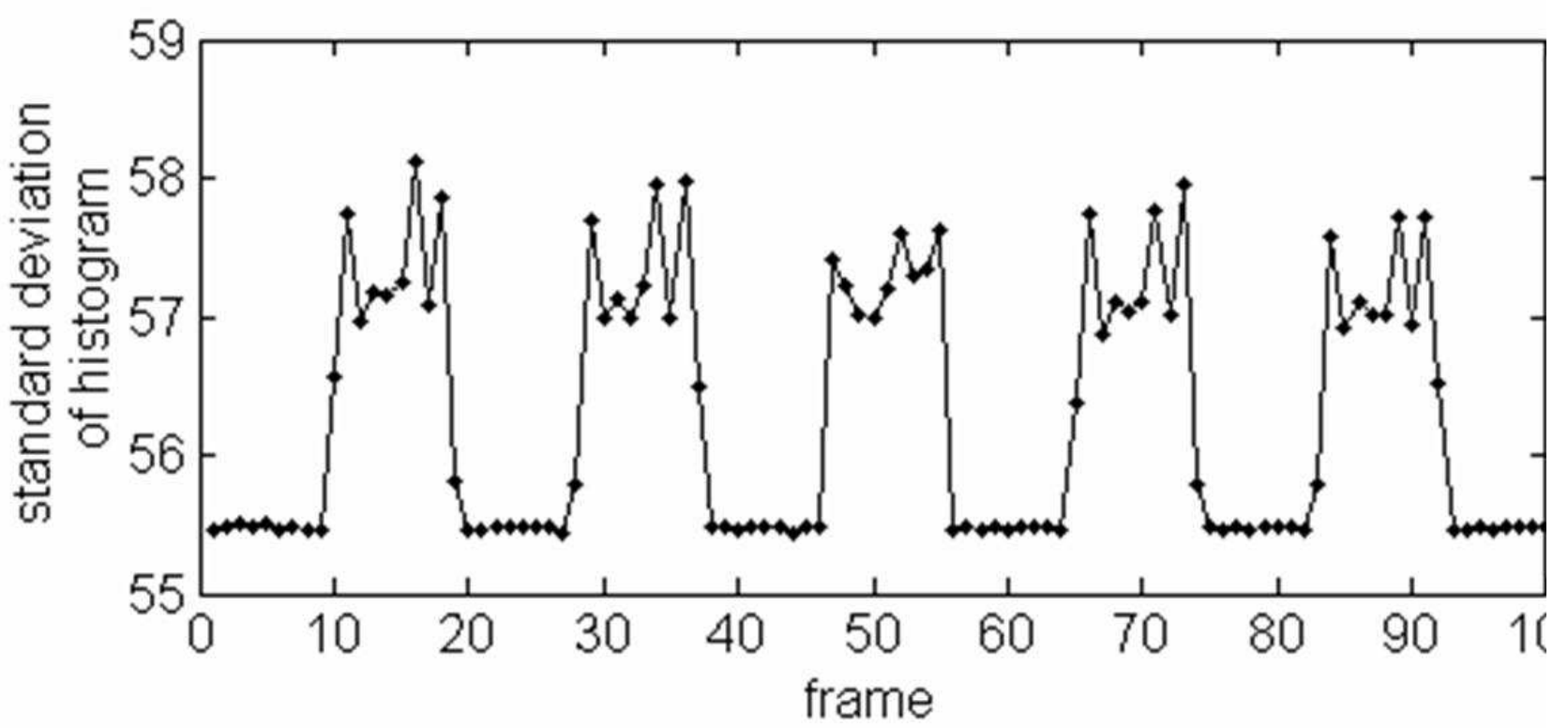

Figure 3 


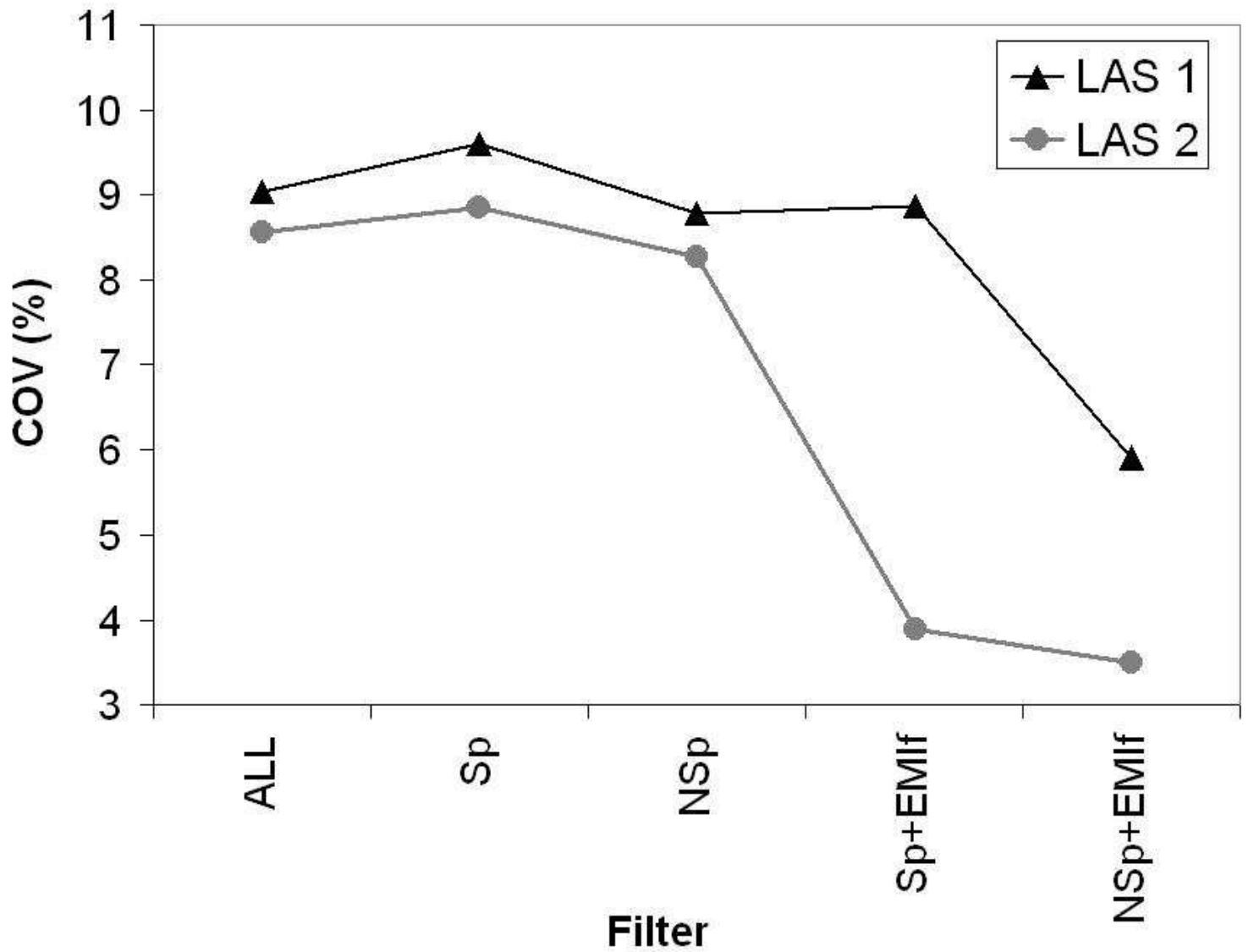

Figure 4 

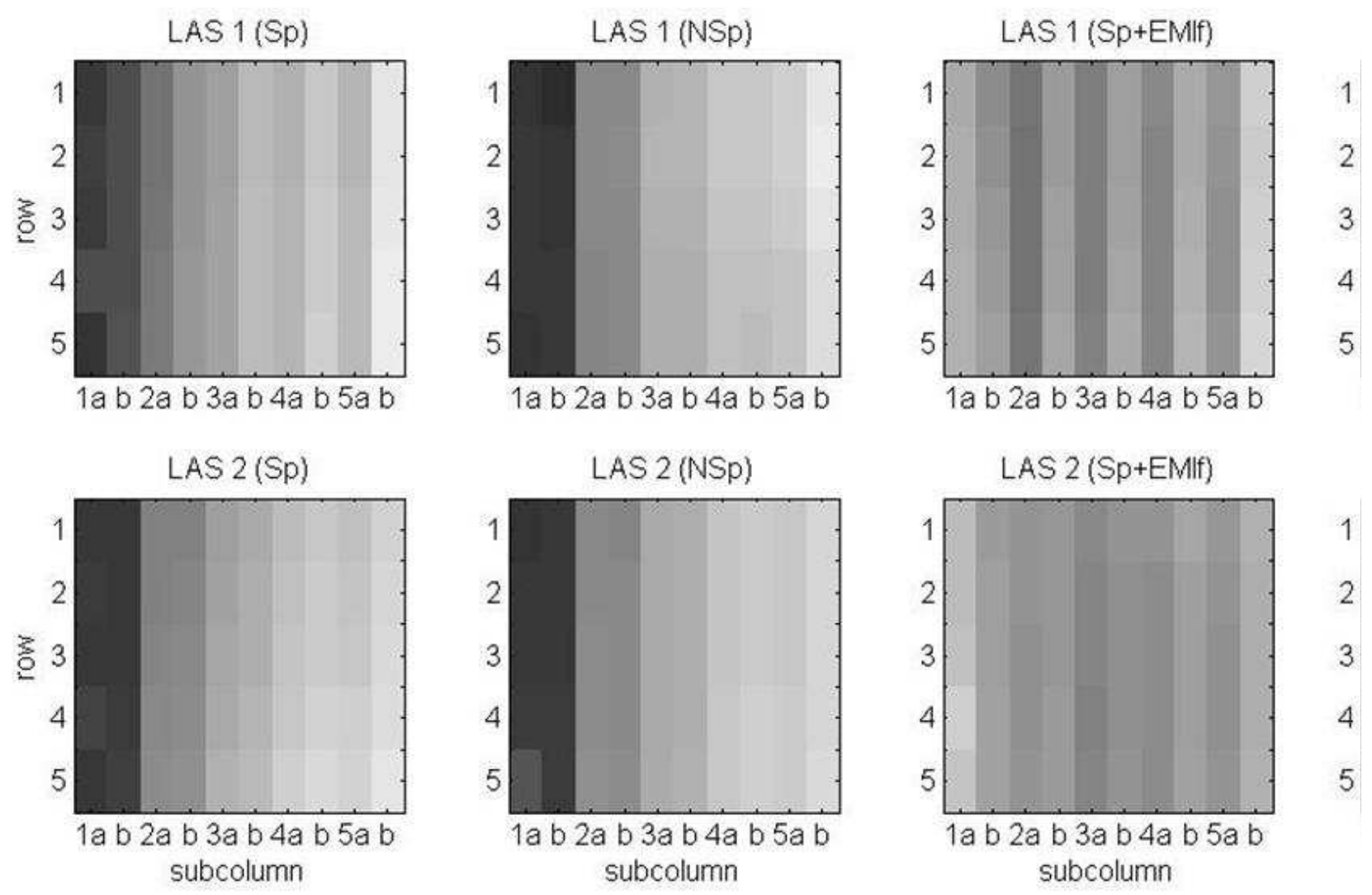

Figure 5 


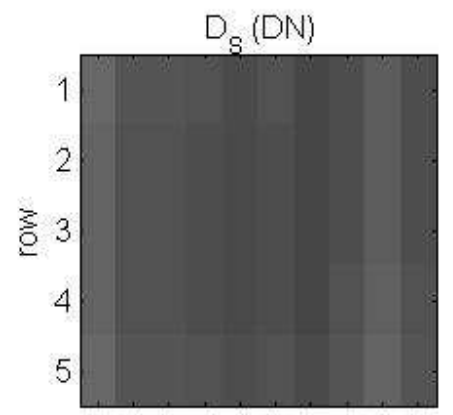

1a b 2a b 3a b 4a b 5a b

$\operatorname{RdN}\left(e^{-}\right)$

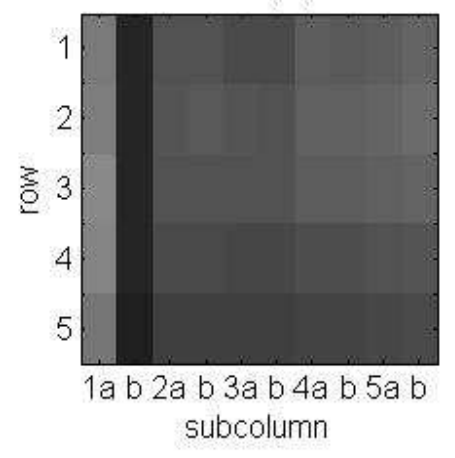

$N\left(e^{-i} / D N\right)$

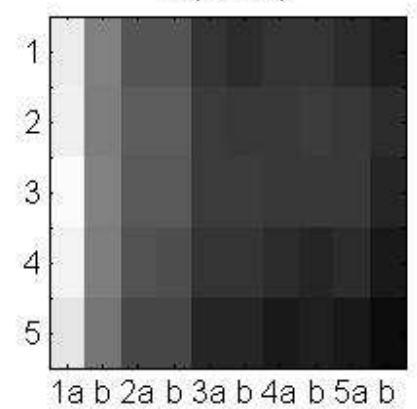

FW $\left(e^{-}\right)$

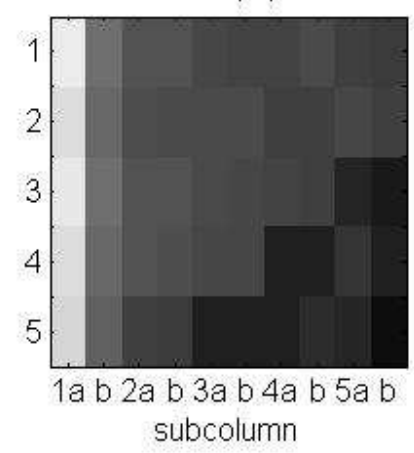

Figure 6

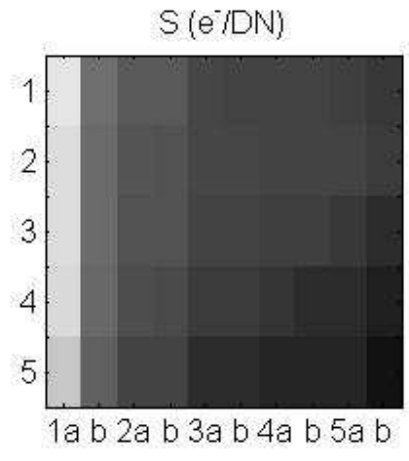

$\%$

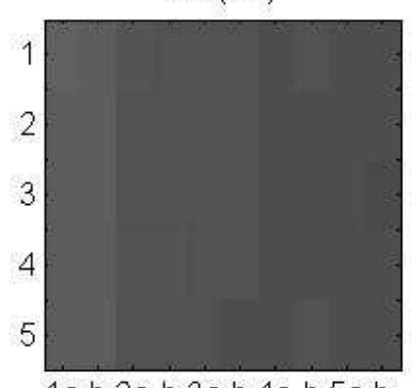
1a b 2a b 3a b 4a b 5a b subcolumn 160 140 120

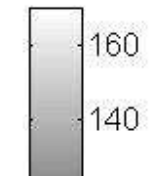

100

80 

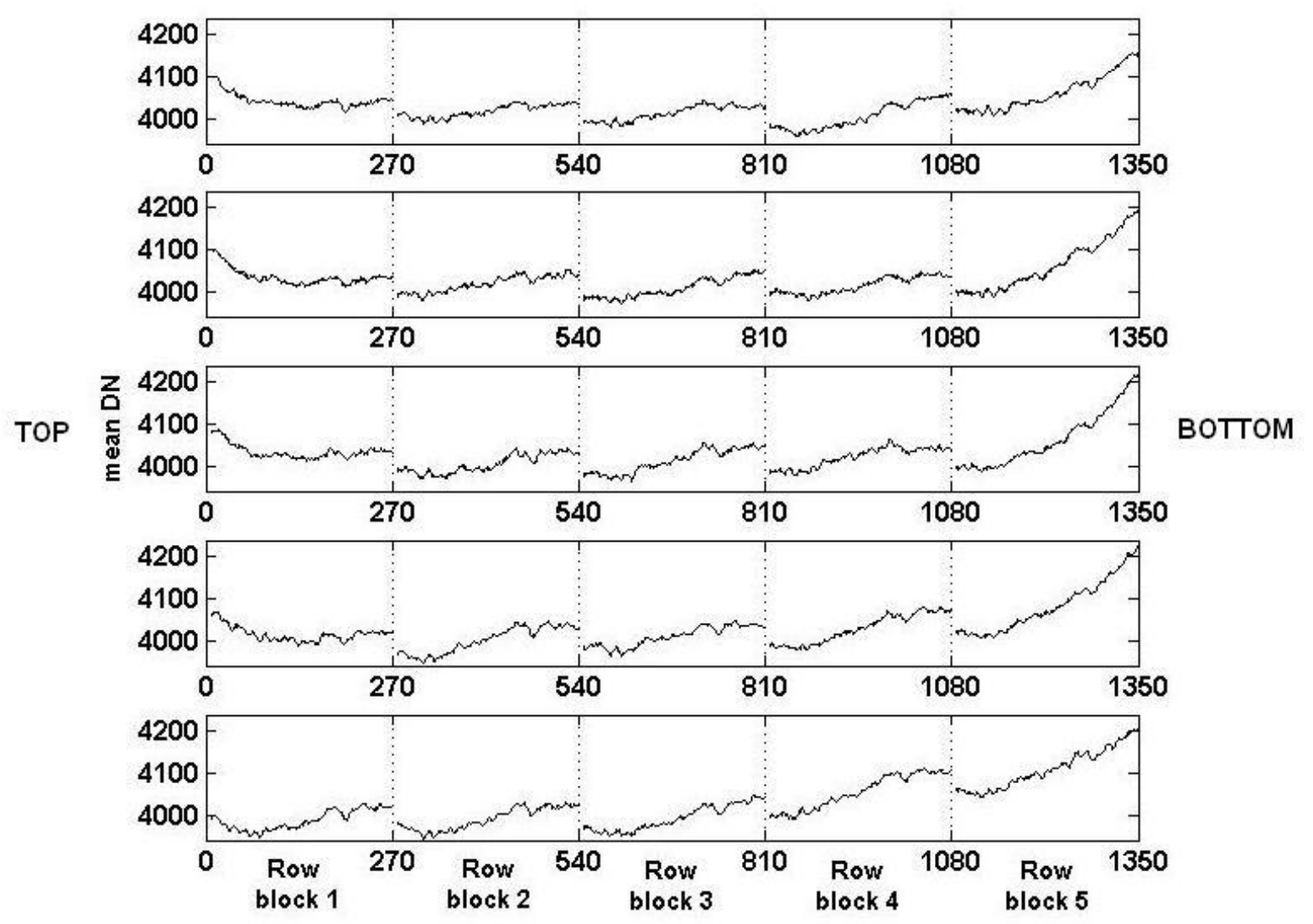

Figure 7 


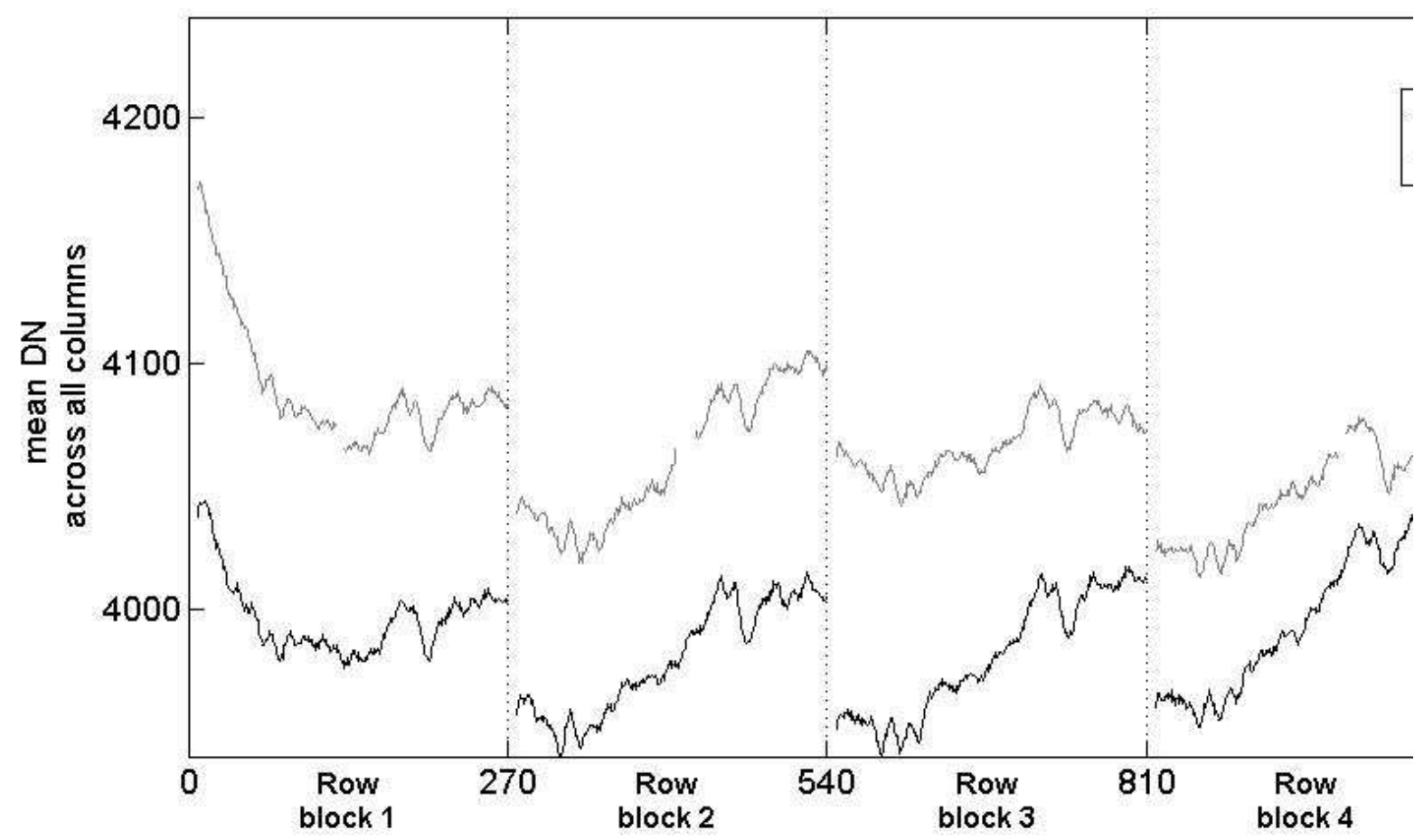

Figure 8

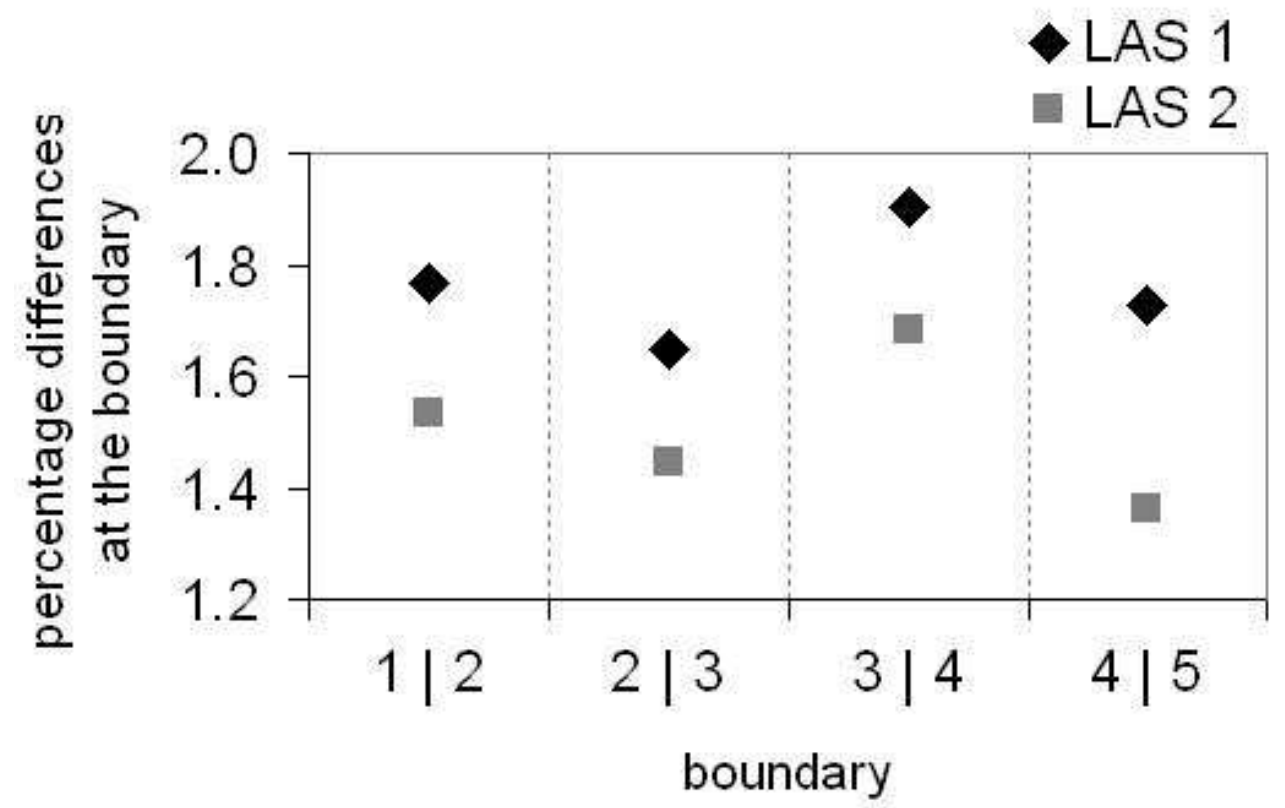

Figure 9 



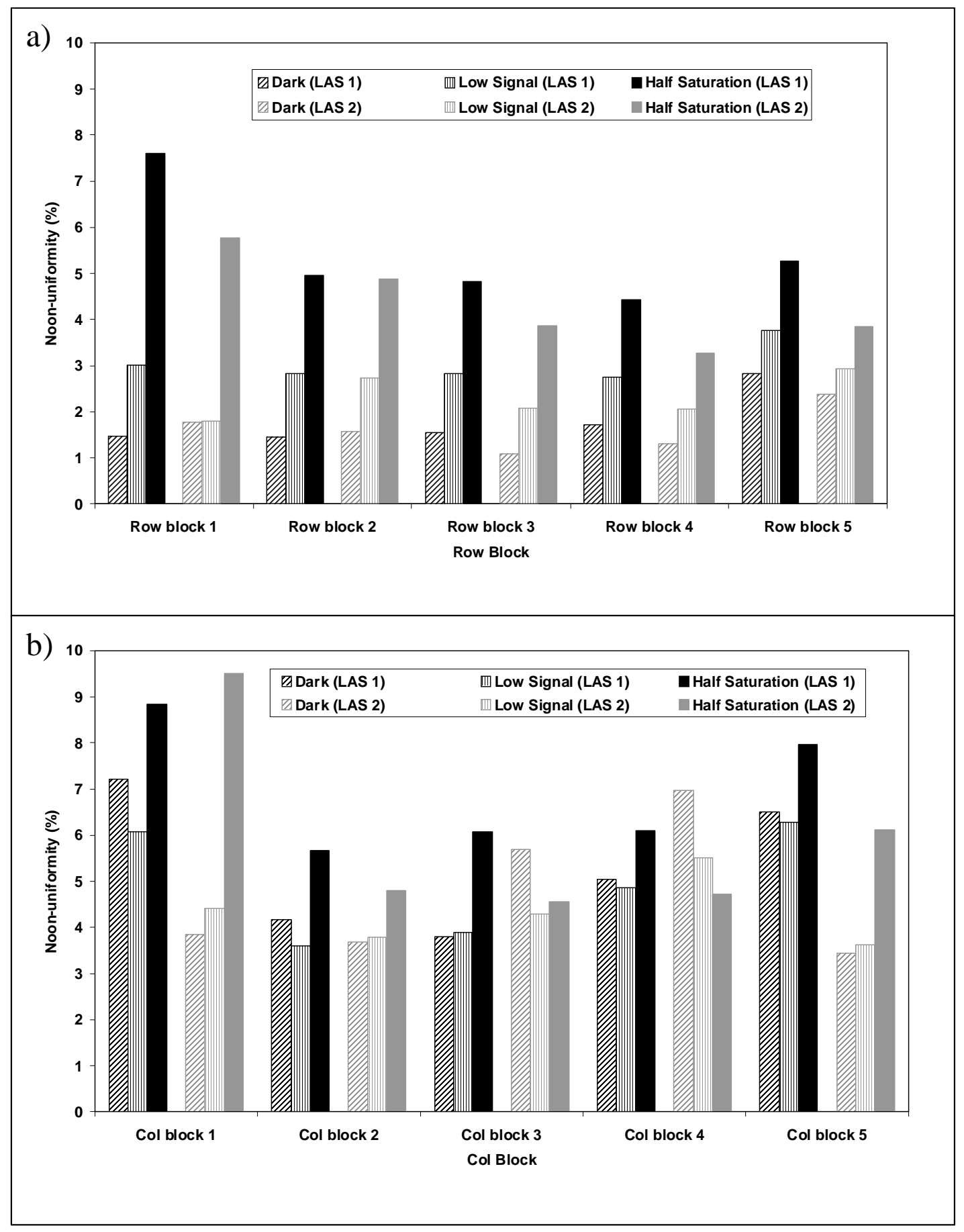

Figure 10 


\section{Figure Captions}

Figure 1: Schematic diagram of LAS architecture. Corner pieces (A), edge pieces (B1, B2, $\mathrm{C} 1$ and $\mathrm{C} 2$ ) and image sensor arrays (D) create a larger array of 25 sensitive blocks and 24 peripheral blocks. Each column sub-block was connected to two ADC channels (shown as ch1 to ch10).

Figure 2: Dark image averaged over 200 frames. Strong columnar variation is seen corresponding to the different sub-column read-out structure. Stitched region row and columns are labelled at the top and right hand side of the image and sub-columns are labelled at the bottom.

Figure 3: Standard deviation of the distribution of pixel values as a function of frame number for sub-column $3 \mathrm{~b}$. SD is high when spiking pixels are present.

Figure 4. Coefficient of variation (COV) between average pixel dark noise in each of the sub-columns for LAS 1 and LAS 2 for the 5 cases described in the text. On the x-axis, 'All' denotes all frames without EMI filtration, 'Sp' denotes spiking frames only without EMI filtration, 'NSp' denotes non-spiking frames only without EMI filtration, 'Sp+EMIf' is spiking frames with EMI filtration and 'NSp+EMIf' denotes spiking frames with EMI filtration.

Figure 5: Maps of variation of mean pixel dark noise for each sub-region expressed as percentage variation from the mean pixel dark noise for the whole sensor for LAS 1 and LAS 2 for 4 of the 5 cases described in the text. On the x-axis, 'Sp' denotes spiking frames only without EMI filtration, 'NSp' denotes non-spiking frames only without EMI filtration, 'Sp+EMIf' is spiking frames with EMI filtration and 'NSp+EMIf' denotes spiking frames with EMI filtration.

Figure 6. Maps of performance parameters averaged for each sub-stitch region and expressed as percentage variation from the mean value. Parameters shown are: dark signal ' $D_{S}$ ' (units DN), noise gain ' $N$ ' (e $\left.e^{-/ D N}\right)$, signal gain ' $S$ ' (e/DN), read noise 'RdN' (e'), full well capacity 'FW' (e'), and dynamic range 'DR' $(\mathrm{dB})$.

Figure 7: Averaged row-to-row profiles across each of the stitched regions for a dark signal image from LAS 1 . Row block 1 denotes the set of rows in the first stitched region etc.

Figure 8: Row-to-row profile averaged across the whole sensor for dark signal images from LAS 1 and LAS 2. The gaps in the plot for LAS2 show the presence of dead lines in this sensor.

Figure 9: Differences at the stitch boundaries for both sensors.

Figure 10: a) Percentage non-uniformity, calculated using eq. 1, as a function of row block number for both sensors at three illumination levels: dark level ('Dark'), low signal and half saturation signal. b) Percentage non-uniformity, calculated using eq. 1, as a function of column block number for both sensors at three illumination levels: dark level ('Dark'), low signal and half saturation signal. 
